\title{
"Strafspiel" und satirische Stilmittel in musikdramatischen Gattungen des frühen 18. Jahrhunderts
}

\author{
LIVIO MARCALETTI, WIEN
}

Die heutige Operngeschichtsschreibung tendiert oft zu einer Vereinfachung der im 18. Jahrhundert vorhandenen Gattungsvielfalt; dementsprechend beschränken sich viele Publikationen über Operngeschichte noch auf eine Dichotomie zwischen Opera seria und Opera buffa. ${ }^{1} \mathrm{Zu}$ einer solchen Zweiteilung passen insbesondere Genera mixta wie etwa Tragicommedia per Musica und Dramma eroicomico nicht, die im 18. Jahrhundert anders zugeordnet wurden. Ein Beispiel einer angemessenen Kategorisierung der Operngattungen ist Johann Mattheson zuzuschreiben: In seinem Traktat Der vollkommene Capellmeister (1739) analysiert er den Charakter der auf die verschiedenen musiktheatralischen Gattungen zutreffenden Melodien. Abgesehen von kleineren Gattungen wie Serenata per Musica oder Pastorale, basiert Matthesons Kategorisierung auf einem dreigeteilten Schema, bei dem man zwischen Trauerspiel (Tragoedia), Lustspiel (Comoedia) und Strafspiel (Satyra) unterscheidet:

\begin{abstract}
"Ist die Absicht eines Sing-Spiels tragisch, so muß sich der Gesang darnach richten, und müssen lauter majestätische, ernsthaffte, klägliche Melodien, nach Befinden der Umstände, dabey eingeführet werden, absonderlich zuletzt. Ist aber das Ende einer Oper comisch und lustig, so kehret man es um, und bedienet sich vornehmlich zu rechter Zeit, freudiger, frölicher und anmuthiger Melodien. Ist endlich der Inhalt satyrisch (wiewol deren wenige seyn werden) so müssen die Sang-Weisen hie und da etwas lächerlich, poßierlich und stachelicht herauskommen. Exempel der ersten Art können etwa aus einem Nero; der zwoten aus einem Jodolet, und der dritten aus einem Don Quixotte genommen werden." ${ }^{2}$
\end{abstract}

Während die Übereinstimmung von Trauerspiel und Dramma per Musica bzw. von Lustspiel und Commedia per Musica naheliegt, ist die Definition von Strafspiel nicht eindeutig. Mattheson zufolge sind Opern satirischen Inhalts nur "wenige", und der Charakter ihrer Melodien "lächerlich", "poßierlich" und "stachelicht". Was bedeuten diese Adjektive allerdings konkret? Wie sind solche Eigenschaften bei der Vertonung einer Arie satirischen Sujets erkennbar?

Der Verständlichkeit halber nennt Mattheson als einziges Beispiel für das Strafspiel die Oper Don Chisciotte in Sierra Morena (Wien 1719), des Librettisten Pietro Pariati und des Komponisten Francesco Bartolomeo Conti. 1722 wurde sie in Hamburg mit direkter Beteiligung Matthesons

1 Rezente Veröffentlichungen stellen einen ersten Versuch dar, ein vielfältigeres Bild der musikdramatischen Gattungen im 17. und 18. Jahrhundert wiederzugeben, wie etwa in Hinsicht auf den Wiener Kaiserhof von Leopold I., vgl. Sebastian Biesold und Philipp Kreisig, „Jenseits des Gattungsdenkens: Musikdramatische Werke und Herrschaft im Alten Reich", in: Musikwissenschaft: die Teildisziplinen im Dialog, Mainz 2016, S. 1-14.

2 Johann Mattheson, Der vollkommene Capellmeister, Hamburg 1739, S. 219. 
aufgeführt, der die deutschen Rezitativtexte vertonte und die Arien überarbeitete. ${ }^{3}$ Es handelt sich um eine Tragikomödie (Tragicommedia per Musica), eine Operngattung, welche die Karnevalsaison am Wiener Kaiserhof im frühen 18. Jahrhundert charakterisierte. ${ }^{4}$ Die Tragicommedia per Musica mischt ernste und komische Figuren in einer Handlung, die in den traditionellen, glücklichen Ausgang mündet. Anders als in den Drammi per Musica der Zeit, die noch eine Zusammenstellung einer ernsten und einer komischen Handlung vorsahen, involviert die Tragicommedia per Musica auch Ritter, KönigInnen, Philosophen usw., die verspottet und lächerlich gemacht werden dürfen. In Alessandro in Sidone (1721) werben zwei griechische Philosophen um dieselbe Frau und streiten mit schlüpfrigen Beleidigungen; ${ }^{5}$ in Penelope (1724) verstellt sich der Held Odysseus als Diener, um die Treue seiner Gattin Penelope zu überprüfen, und beweist sich eher als übermäßig eifersüchtiger Liebhaber denn als kluger Held. ${ }^{6}$

Mattheson zufolge hänge diese Tragicommedia per Musica mit dem Strafspiel zusammen, das übrigens nie als Gattungsbezeichnung in Libretti oder Partituren und kaum im zeitgenössischen literarischen Diskurs auftaucht. Dafür soll auf Martin Opitz' Erläuterung der Eigenschaften und der Ziele der Satire (Buch der deutschen Poeterey, 1624) zurückgegriffen werden:

"Zue einer Satyra gehören zwey dinge: die lehre von gueten sitten vnd ehrbaren wandel, vnd höffliche reden vnd schertzworte. Jhr vornemstes aber vnd gleichsam als die seele ist, die harte verweisung der laster vnd anmahnung zue der tugend: welches zue vollbringen sie mit allerley stachligen vnd spitzfindigen reden, wie mit scharffen pfeilen, vmb sich scheußt. Vnd haben alle Satyrische scribenten zum gebrauche, das sie vngeschewet sich vor feinde aller laster angeben, vnd jhrer besten freunde ja jhrer selbst auch nicht verschonen, damit sie nur andere bestechen mögen: wie es denn alle drey Horatius, Juuenalis vnnd Persius meisterlich an den tag gegeben." ${ }^{7}$

Von den Modellen der lateinischen Literatur ausgehend, hebt diese Definition das moralische Ziel einer Satire hervor, bei der die Tugend gepriesen und das Laster gestraft wird. Opitz bezieht sich allerdings auf nicht-dramatische Gedichte: Horatius, Juvenalis und Persius waren keine Dramatiker, und er nennt in demselben Kapitel das Epigramma "eine kurtze Satyra". Hingegen verknüpft Mattheson den Begriff Satire mit der Oper und mit dem Terminus "Strafspiel". Die Etymologie des letzteren Wortes verweist auf die vorbildliche Bestrafung der Untugend eines Bösewichts im Kontext eines "Spiels" - womit das Theater einbezogen wird. Die Philosophen, die wegen ihrer Inkohärenz lächerlich aussehen; Odysseus, dessen Eifersucht übertrieben

3 Siehe Konstantin Hirschmann, Die Tragicommedia Pietro Pariatis und Francesco Contis und ihre Rezeption in Norddeutschland am Beispiel des Don Chisciotte in Sierra Morena, Masterarbeit, Wien 2016, S. 116-28.

4 Siehe u. a. Claudia Michels, Karnevalsoper am Hofe Kaiser Karls VI.: Kunst zwischen Repräsentation und Amusement, Wien 2019.

5 Die Tradition der "Philosophenkomödien” stammt aus den Libretti von Nicolò Minato für den Fasching am Wiener Kaiserhof. Siehe u. a. Herbert Seifert, Die Oper am Wiener Kaiserhof im 17. Jahrhundert, Tutzing 1985, S. 248-262; und Alfred Noe, "I filosofi nei libretti di Nicolò Minato", in: La filosofia e la sua storia. Studi in onore di Gregorio Piaia, Bd. 1, hrsg. von Marco Forlivesi, Padua 2017, S. 261-276.

6 Livio Marcaletti, „Die keusche Penelope und der zornige Odysseus: Kursieren einer tragikomischen Opernhandlung zwischen Wien und Norditalien", in: Musicologica Brunensia 2018 (Supplementum), S. 83-95.

7 Martin Opitz, Buch von der Deutschen Poeterey, Breslau 1624, Nachdr. Stuttgart 1970, S. 28. 
ist; Don Chisciotte, der nicht wegen seiner Untugend, aber als verrückte Parodie eines wandernden Ritters verprügelt und verspottet wird: Sie sind alle Beispiele von "bestraften" Figuren, die als Protagonisten eines Strafspiels fungieren können.

Soweit zur Gattungszuordnung aus literarischer Perspektive. Welche ist nun die musikalische Spezifik der gemischten Operngattungen? Die bisherigen Studien haben tragikomische und satirische kompositorische Elemente vor allem in der instrumentalen Musik von Georg Philipp Telemann zum Thema. In Music for a Mixed Taste hat der nordamerikanische Musikwissenschaftler Steven Zohn den polnischen Stil mehrerer Instrumentalwerke Telemanns mit folgenden Ausführungen von Johann Adolph Scheibe in Zusammenhang gebracht:

„Wir kommen nunmehro auf die Musikart der Pohlen, oder auf den so genannten pohlnischen Styl [...] Der berühmte Hr. Telemann hat ihn am ersten in Schwang gemacht, und uns durch die schönsten Proben dargethan, wie schön dieser Musikstyl ist, wenn sie in seiner Vollkommenheit ausgeübet wird...Insgemein ist diese Schreibart zwar lustig, dennoch aber von großer Ernsthaftigkeit. Mann kann sich auch derselben zu satyrischen Sachen sehr bequem bedienen. Sie scheint fast von sich selbst zu spotten; insonderheit wird sie sich zu einer rect ernsthaften und bittern Satire schicken." ${ }^{8}$

Die "ernsthaft[e] und bitter[e] Satire", mit welcher Scheibe den "sogenannten pohlnischen Styl" verbindet, ist eine oxymoronische Mischung vom "Lustigen" und "Ernsthaften". Ähnliche Bezeichnungen wählt Telemann selbst für einige Werke aus, wie etwa den Ausdruck "die lustige polnische Ernsthaftigkeit" beim Lied "Sanfter Schlaf" (TWVW 25:63) ${ }^{9}$. Bei seiner Analyse erkennt Zohn bestimmte den polnischen Stil kennzeichnende Stilelemente und Topoi, wie etwa den Orchestereinklang, die unübliche Verwendung musikalischer Akzente, welche den konventionellen metrischen Schemata der Tanzsätze widerspricht, oder auch übertriebene Wiederholung rhythmischer und melodischer Figuren, usw.

Diese Ausführungen können mit denen des Literaturwissenschaftlers Gérard Genette fruchtbar in Verbindung gesetzt werden, der in Palimpseste unterschiedliche Arten der Parodie analysiert hat und sich überschneidende Beziehungen von Sujet und Stil mit den Kategorien „burlesker Travestie" und tatsächlicher Parodie folgendermaßen beschreibt:

„Die burleske Travestie modifiziert also den Stil und beläßt das Thema; umgekehrt modifiziert die "Parodie' das Thema und beläßt den Stil, was auf zwei Wegen bewerkstelligt werden kann: entweder es wird der vornehme Text beibehalten und so wörtlich wie möglich auf ein vulgäres (reales und aktuelles) Thema angewandt: dies ist die Parodie im engeren Sinn [...]; oder aber es entsteht durch stilistische Nachahmung ein neuer, vornehmer Text, der auf ein vulgäres Thema bezogen wird: das komisch-heroische Pastiche." ${ }^{10}$

Das Satirische entsteht aus der Inkongruenz hoher stilistischer Elemente, die auf niedrige Rollen angewendet werden, oder umgekehrt niedriger Stilelemente, die gegen die Erwartungen

8 Johann Adolph Scheibe, Critischer Musikus, Leipzig 1745, S. 149.

9 Steven Zohn, Music for a Mixed Taste. Style, Genre, and Meaning in Telemann's Instrumental Works, Oxford 2015, S. 487.

10 Gérard Genette, Wolfram Bayer und Dieter Hornig (Übers.), Palimpseste: die Literatur auf zweiter Stufe, Frankfurt 2012, S. 36. 
auf hohe Figuren angewendet werden. Wie lassen sich in Vokalwerken Elemente satirischer und komischer Musik voneinander unterscheiden? In Bezug auf Contis Don Chisciotte hat sich Konstantin Hirschmann als einer der ersten damit beschäftigt, wie Matthesons Kategorisierung sich auf Contis Don Chisciotte anwenden lässt, deren musikalische Ideen "auf dem blossen Papier fast eben die ergetzliche Wirckung mit sich [führten], als ob man mit Augen allerley lächerliche, lebendige Posituren vor sich sähe". ${ }^{11}$ Von dieser Behauptung ausgehend, zeigt Hirschmann wie Contis Vertonung mit den Konventionen des ernsten Dramma per Musica spielt, um komische Effekte auszuüben. Im ersten Akt singt der adlige, zum Wahnsinn aus Eifersucht getrieben Cardenio eine scheinbar konventionelle Wutarie, die bestimmte motivische Elemente zur Parodie verwandeln, wie etwa die Dezimsprünge der Violinen und die Circoli mezzi von Bratsche und Cello im Unisono: ${ }^{12}$

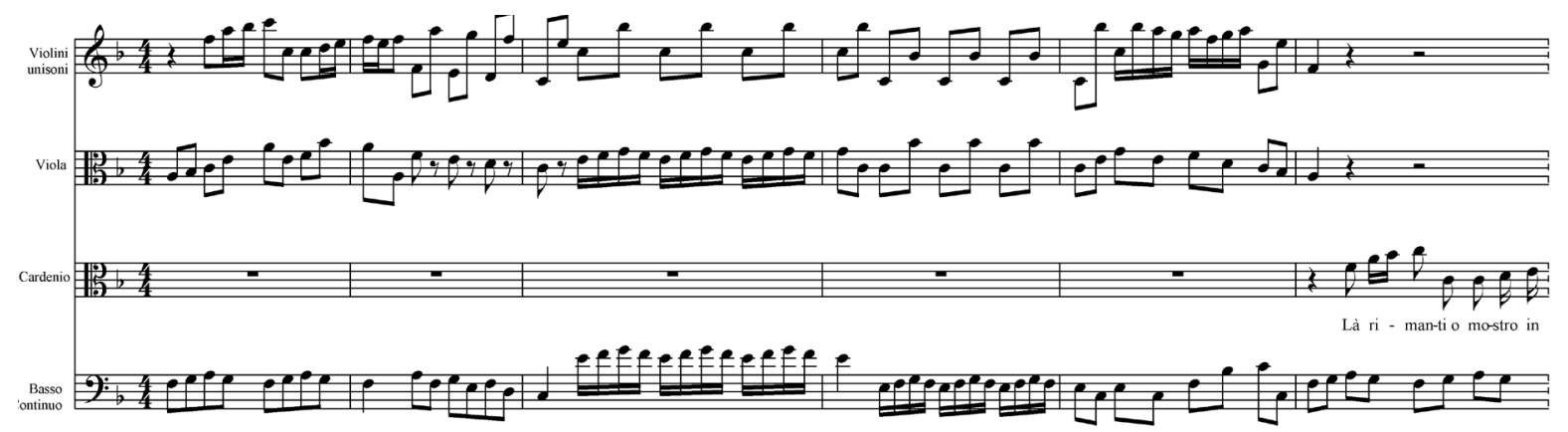

Notenbeispiel 1: 1: Francesco Bartolomeo Conti, "Là rimanti o mostro infido" aus Don Chisciotte in Sierra Morena, T. 1-6.

Die übertriebene Wiederholung bestimmter melodischer Figuren, in Zusammenhang mit einer eindeutig satirischen dramaturgischen Situation, ist ein Stilmittel, das auch bei anderen Komponisten und anderen vokaldramatischen Gattungen seinen Platz findet. Eine erstaunliche Ähnlichkeit findet man in einem deutlich anderen Kontext, und zwar in einer weltlichen Kantate von Johann Sebastian Bach. Bei Geschwinde, ihr wirbelnden Winde (BWV 201) handelt es sich um den Musikwettstreit zwischen Pan und Apollo, eine Handlung, die tragikomische Züge innehat: Wenn Mida Pan als Gewinner des Streits ankündigt, bestraft Apollon ihn durch Eselsohren. Die Arie, in der Mida die Ankündigung gibt, enthält zu diesem satirischen Zweck nicht nur eine sehr ähnliche Wiederholung von Circoli mezzi wie bei der obengenannten Arie von Conti, sondern auch eine Nachahmung des Eselschreies als Vorahnung der Strafe, die Mida erwartet:

11 Mattheson, Der vollkommene Capellmeister, S. 40; siehe auch Hirschmann, Die Tragicommedia Pietro Pariatis und Francesco Contis, S. 76.

12 Hirschmann, Die Tragicommedia Pietro Pariatis und Francesco Contis, S. 81. 


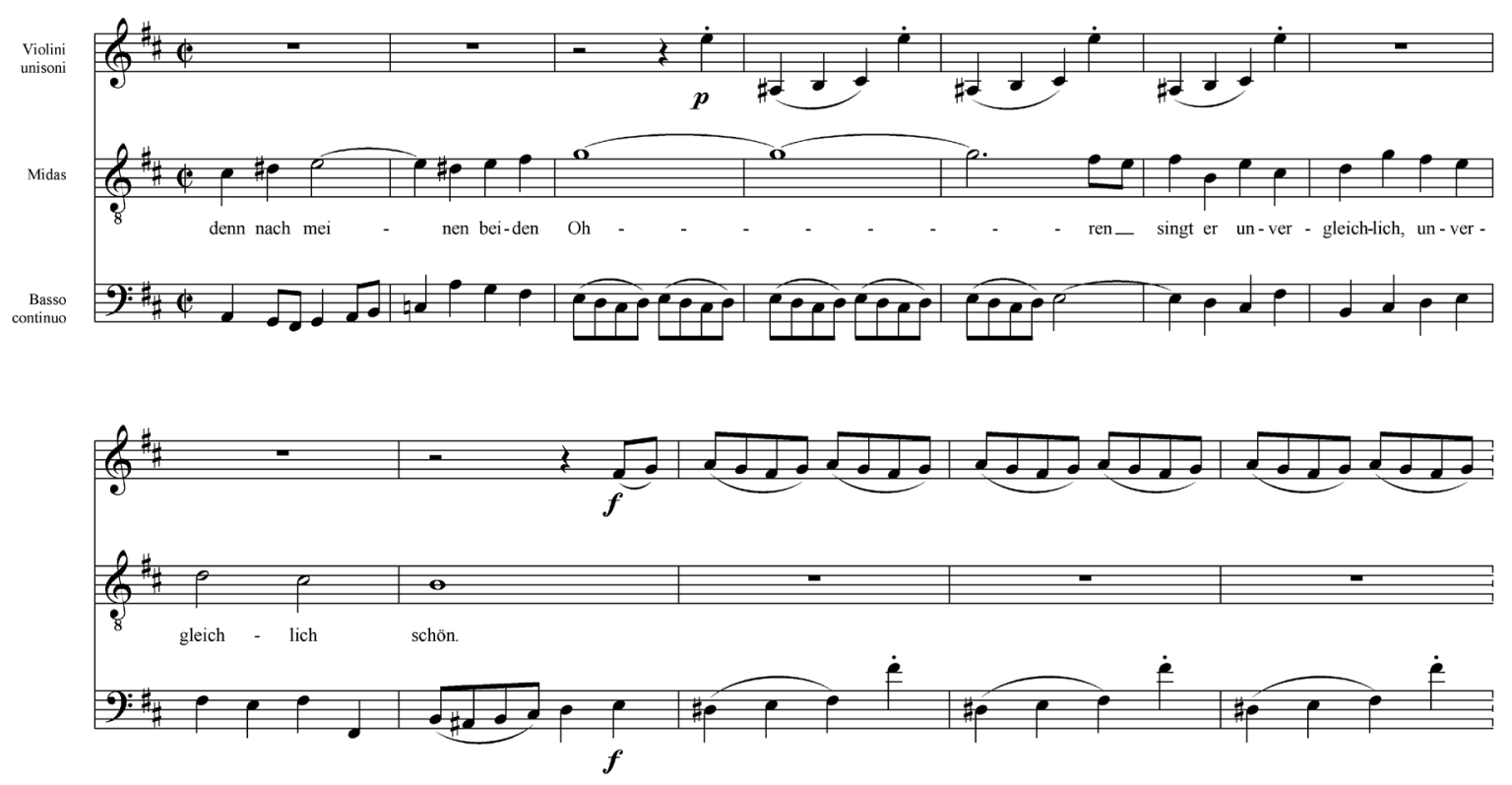

Notenbeispiel. 2: Johann Sebastian Bach, "Pan ist Meister, lasst ihn gehn”, T. 108-119 aus Geschwinde, ihr wirbelnden Winde (BWV 201).

Diese Anwendung besonderer Stilmittel ist m. E. eher als satirisch denn als komisch zu kennzeichnen, weil es sich um eine „burleske Travestie" handelt: Die Würde eines Königs wird durch den Vergleich mit einem Tier beschädigt. Für solche dramatischen Situationen hatte Georg Friedrich Händel eine besondere Vorliebe, die er sowohl in einigen Drammi per Musica als auch in seinen englischen Oratorien zeigt. Die Verspottung einer Figur des Dramas durch eine andere ist eines der interessantesten Beispiele, bei denen Händel satirische Effekte durch übertriebene Wiederholung erzeugt. Dies soll an zwei Beispielen gezeigt werden, die beide die Verspottung eines Mannes durch eine Frau, die ihn für "verweichlicht" hält, darstellen. Hercules (1745), ein tragisches Oratorium nach den Vorlagen von Sophokles' Die Trachinierinnen und Ovids Metamorphosen, schildert die Eifersucht von Dejanira, der Gattin des Herkules, ${ }^{13}$ die ihren Gatten einer Liaison mit der Gefangenen lole verdächtigt. Dejanira verspottet Herkules, ohne inm beißende Satire in der Arie „Resign thy Club" zu ersparen: ${ }^{14}$ Sie fordert ihn auf, die Waffen abzulegen und sich weiblichen Tätigkeiten und der Liebe zu widmen, indem sie den folgenden Text singt:

\footnotetext{
„Resign thy Club and Lion's Spoils,

And fly from War to female Toils;

For the glitt'ring Sword and Shield
}

13 Ruth Smith, Handel's Oratorios and Eighteenth-century Thought, Cambridge 1995, S. 56-60.

14 David Ross Hurley, "Dejanira, Omphale and the Emasculation of Hercules: Allusion and Ambiguity in Handel”, in: Cambridge Opera Journal 11 (1999), S. 199-213. 
The Spindle and the Distaff wield:

Thund'ring Mars no more shall arm thee;

Glory's Call no more shall warm thee;

Venus and her whining Boy

Shall all thy wanton Hours employ."

„Leg ab die Keul' und Löwenhaut

Und flieh vom Kampf zu Weibertand,

Für den blinkenden Speer und Schild

Nimm Rocken und Spindel du zur Hand:

Nicht mehr lockt der Gott des Krieges,

Nicht mehr dich der Ruhm des Sieges,

Nur der Liebe kind'scher Gott,

Er treibt mit deinem Herzen Spott.."15

In dem A-Teil klingt die Erwähnung der "weiblichen" Werkzeuge ("the spindle and the distaff wield") durch die häufige Wiederholung einer großen Sekunde fast hypnotisch, eine sehr zutreffende Vertonung der besessenen Eifersucht Dejaniras sowie eine tonmalerische Darstellung der repetitiven Arbeit am Webstuhl. Die bissige Stimmung der Arie ist explizit seit dem anfänglichen Ritornell, wo die Violine - und daraufhin die Stimme - eine Melodie mit wiederholten Noten in Spiccato, zahlreichen Doppelvorschlägen und übertriebenem Wechsel zweier Töne spielen:

3a)

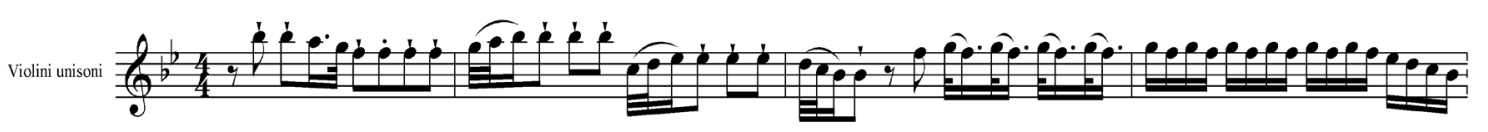

3b)
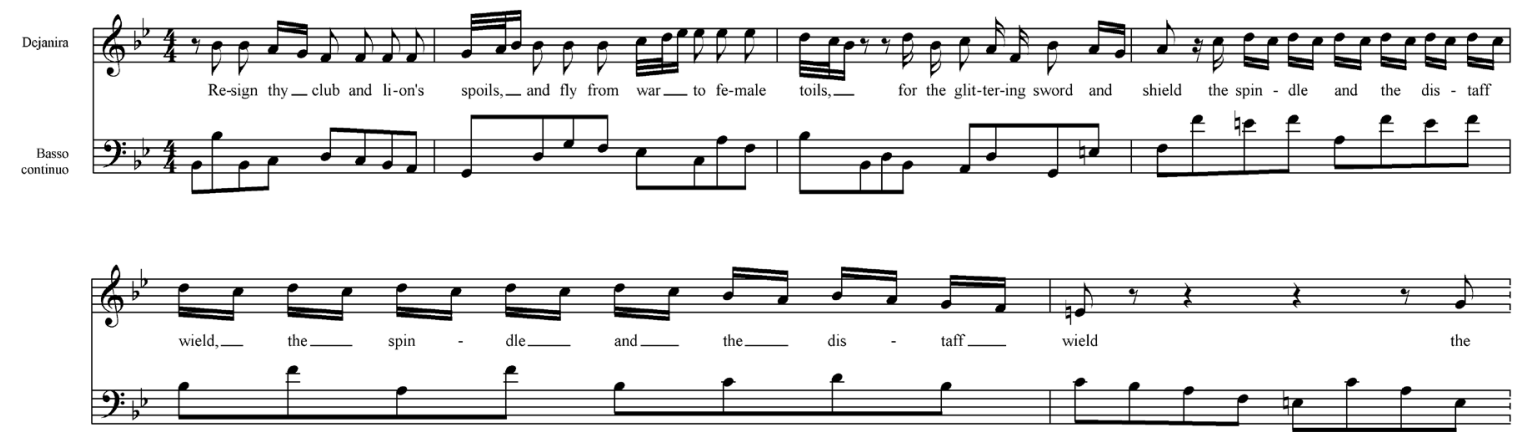

Notenbeispiel 3a und b: Georg Friedrich Händel, „Resign thy Club”, T. 1-4 und 13-18 aus Hercules (HWV 60).

15 Deutsche Übersetzung von Friedrich Chrysander, vgl. Georg Friedrich Händel's Werke, Bd. 4: Hercules, hrsg. von Georg Friedrich Händel und Friedrich Chrysander, Leipzig 1859, S. 163-7. Originaltext auf Englisch n. Chrysanders Edition. 
Die Spiccati und die nacheinander gesetzten Ornamente zeigen eine erstaunliche Ähnlichkeit mit einer anderen ,spöttischen' Arie aus Giulio Cesare in Egitto (1724). In ihrer ersten Arie spricht Cleopatra ihren Bruder in einer vergleichbaren dramaturgischen Situation an, wobei sie seinem Bruder Tolomeo den Thron streitig macht und ihn sarkastisch auffordert, sich der Liebe statt der Politik zu widmen:

„Non disperar, chi sa?

Se al regno non l'avrai,

Avrai sorte in amor.

Mirando una beltà

In essa troverai

A consolar un cor."

„Verzweifle nicht, wer weiß?

Wenn du kein Glück beim Regieren hast,

wirst du es bei der Liebe haben.

Beim Anschauen einer Schönheit

kannst du

dein Herz trösten.. ${ }^{16}$
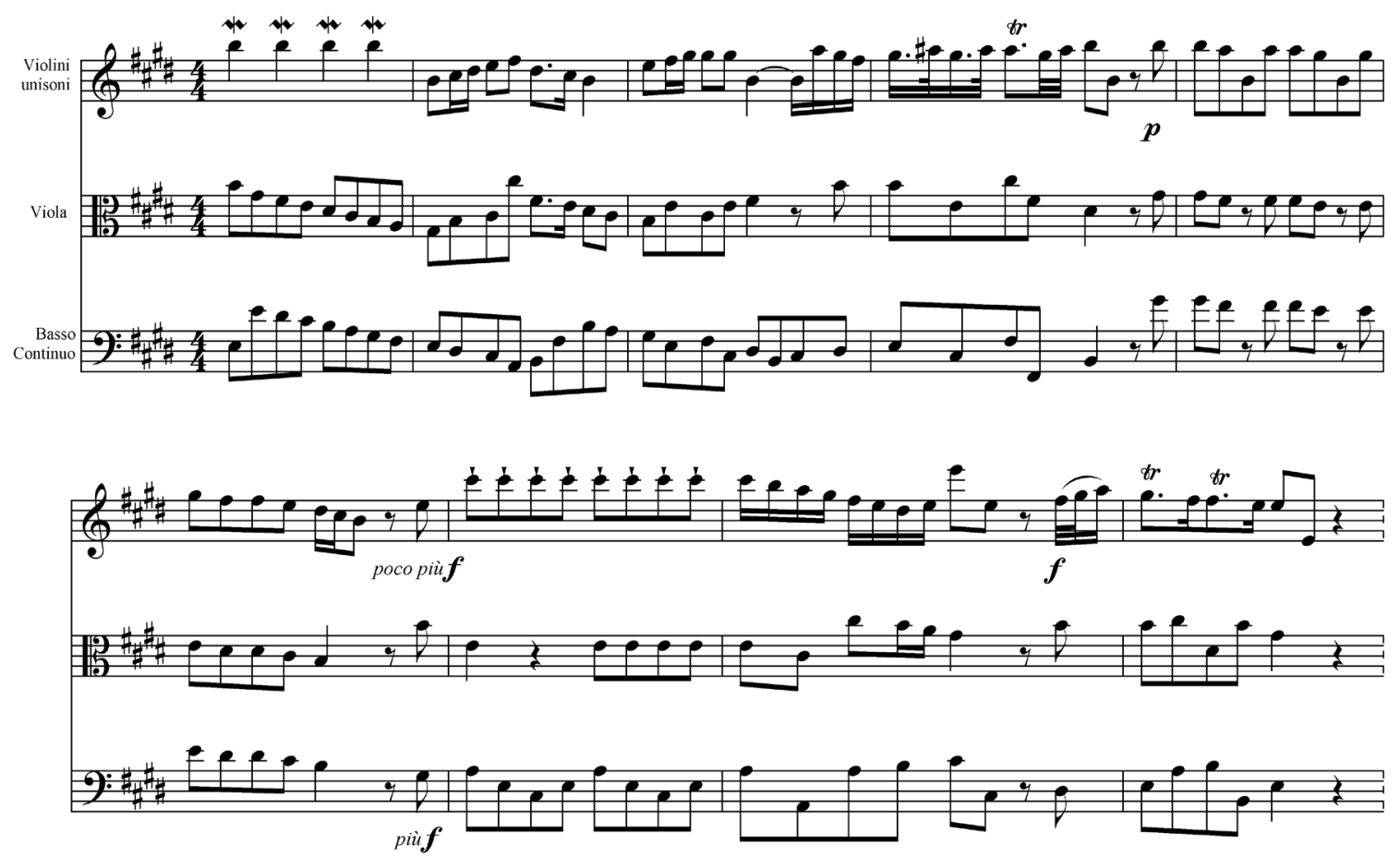

Notenbeispiel 4: Georg Friedrich Händel, „Non disperar, chi sa”, aus Giulio Cesare in Egitto (HWV 17).

Wiederum übt die übertriebene Wiederholung von Spiccato-Tönen in Zusammenhang mit einem spöttischen Text eine mäßige satirische Wirkung aus. Darüber wird auch in späteren

16 Übersetzung L.M. 
musikästhetischen Werken diskutiert, wie in Bernard de La Cépèdes La Poëtique de la musique (1785). In diesem Traktat über die Komposition verschiedener französischer Operngattungen widmet der französische Naturforscher und Opernkomponist der Comédie lyrique ein kurzes Kapitel. In einem Satz darüber, wie Komponisten ihre Musik "komisch" und "burlesk" machen können, äußert sich der französische Musikästhetiker folgendermaßen: „Premièrement, le compositeur pourra rendre sa musique comique, \& même burlesque, en répétant plusieurs fois de suite, \& sans interruption, quelques passages assez saillans $\&$ assez courts pour qu'on puisse, en quelque sorte, compter toutes leurs répétitions". ${ }^{17}$ Es muss aber an dieser Stelle wiederum festgestellt werden, dass der Unterschied zwischen "Komischem" und „Burleskem" nicht ausschließlich durch die Musik bemerkbar ist, sondern anhand der gesamten dramaturgischen Situation: Welche Figur die Arie singt, in welchem Kontext, in welchem Sprachregister gesprochen wird usw.

Die mehrfache Wiederholung eines Motivs bzw. einer Passage ist nicht das einzige Mittel, über das ein Komponist verfügte, um in einem „satirischen" Stil zu schreiben. Händel bediente sich u. a. des häufigen und unerwarteten Wechsels von Dur-Moll, etwa in Zusammenhang mit einem ungewöhnlichen melodischen Duktus. Diese Beispiele findet man in denen Opern Händels, die aus der gattungsgeschichtlichen Perspektive schwer zuzuordnen sind, wie etwa Flavio (HWV 16). Winton Dean zufolge ist Flavio in modernen Zeiten wenig aufgeführt und unterschätzt worden, auch weil seine Gattungszugehörigkeit nicht verstanden worden ist. ${ }^{18}$ Das ungewöhnliche Libretto ist eine Überarbeitung von Flavio Cuniberto von Matteo Noris (Uraufführung 1682, als Vorlage wird jedoch die Fassung aus Rom von 1696 verwendet), das seinerseits auf der Tragikomödie Le Cid von Pierre Corneille basiert. In der Lombardei des frühen Mittelalters regiert der langobardische König Flavio Cuniberto (Flavius Cunincpertus) mithilfe seiner Ratgeber Ugone und Lotario. Guido (Sohn von Ugone) und Emilia (Tochter von Lotario) bereiten sich für ihre Hochzeit vor, bis ihre Väter um die Führung des langobardischen Heers in Britannien streiten und Lotario Ugone ohrfeigt. Um ihn zu rächen, fordert Emilio Lotario zum Duell und tötet ihn. Die verzweifelte Emilia verlangt Gerechtigkeit vor dem König, bevor sie sich zum Schluss mit ihrem Liebhaber versöhnt. Die tragischen Ereignisse der Handlung werden mit einer Prise Satire behandelt: Grund des Duells ist eine Ohrfeige, die eine übertriebene Reaktion auslöst; Flavio ist die Karikatur eines Königs, denn er agiert nur im Interesse seiner Liebe für Deodata, Guidos Schwester, und will infolgedessen ihren Vater nach Britannien schicken. Winton Dean bezeichnet diese Oper deswegen als "anti-heroic", denn „while not denying the heroic ideal [...] they poke fun at the convention by stressing its ridiculous as well as its serious

17 Bernard Germain Etienne de La Ville sur Illon de La Cépède, La Poëtique de la musique, Paris 1785, S. 282. Übersetzung L.M.: „Zuerst kann der Komponist seine Musik komisch und sogar burlesk machen, indem er mehrmals nacheinander und ohne Unterbrechung einige sehr prägnante und genügend kurze Passagen wiederholt, so dass alle Wiederholungen genau gezählt werden können".

18 Winton Dean, Handel's operas, 1704-1726, Oxford 1987, S. 465. 
aspects and juxtaposing satire and even parody beside passionate commitment" ${ }^{19}$ Ein interessantes Beispiel ist Emilias Arie am Ende des ersten Aktes mit folgendem Text:

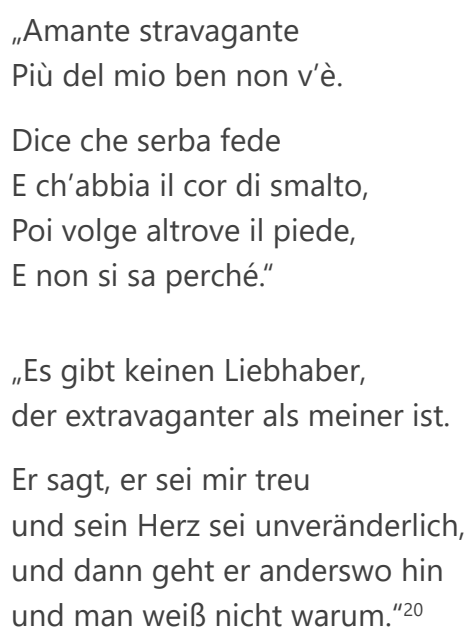

Der Text ist zwar in einem gehobenen Sprachregister geschrieben, aber er weist Ausnahmen auf ("stravagante" ist ein ungewöhnliches Wort für eine Arie eines ernsten Dramma per Musica) und vor allem skizziert er eine für das Ende eines Akts untypische dramaturgische Situation: Ein solcher Streit zwischen Liebhabern könnte unter diesem Aspekt als Satire tragischerer Auseinandersetzungen betrachtet werden. Emilia wirkt durch das "extravagante" Verhalten ihres Verlobten befremdet und belustigt zugleich, was sich auch in der Vertonung widerspiegelt:

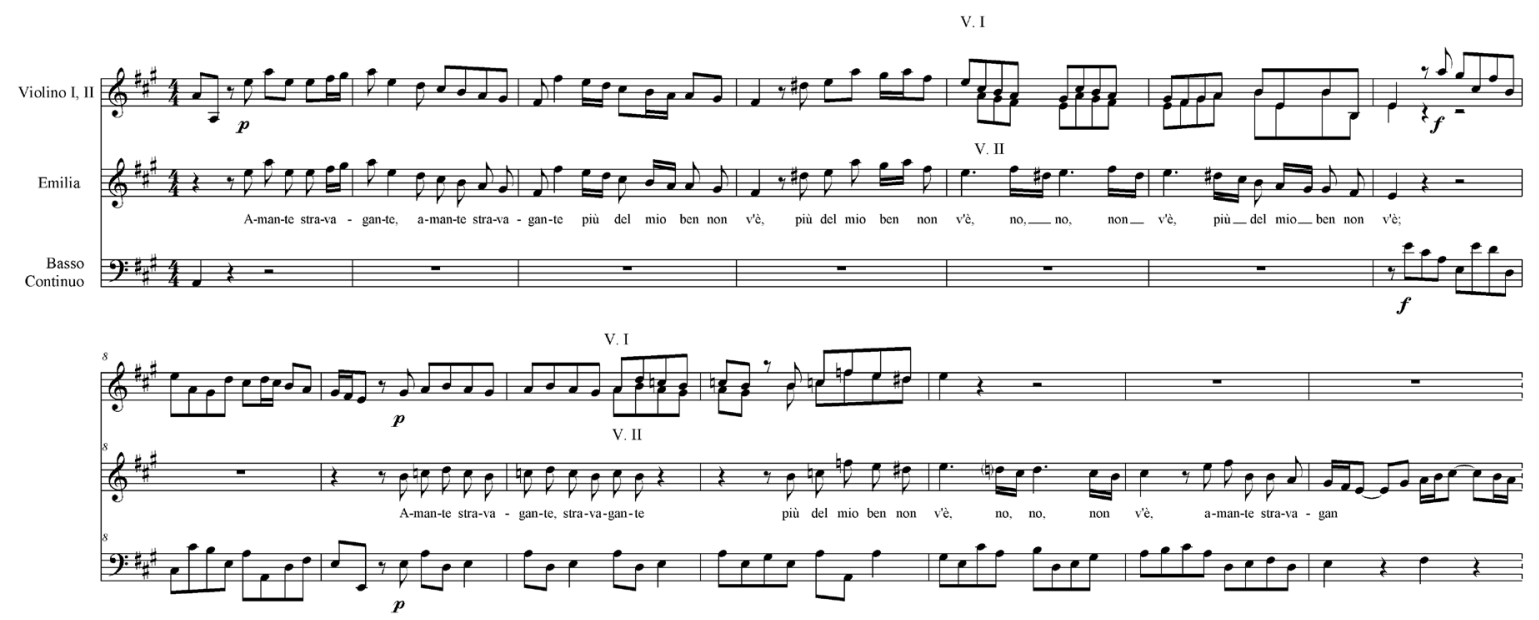

Notenbeispiel 5: Georg Friedrich Händel, „Amante stravagante”, aus Flavio (HWV 16).

Winton Dean hält das Wechselspiel zwischen Dur und Moll für einen Kassandraruf der tragischen Ereignisse, die folgen werden ${ }_{\text {„H}} \mathrm{Handel}$ is careful to touch in the shadows by invoking

19 Winton Dean, "A Handel Tragicomedy”, in: The Musical Times 110, S. 819-22, hier S. 819.

20 Übersetzung L.M. 
the relative and the tonic minor"). ${ }^{21}$ Meines Erachtens wäre jedoch diese Verwechselung als Ausdruck des bizarren Verhaltens von Guido zu betrachten. Kein Zufall, dass Händel eine ähnliche kompositorische Herangehensweise (Verwechslung von Dur und Moll in der Tonika in Zusammenhang mit einer "sorglosen" Melodie mit vielen Intervallsprüngen) für eine andere "satirische" Arie, und zwar "Amor è qual vento" aus Orlando (HWV 31), verfolgte. Wie Reinhard Strohm hervorgehoben hat, ist Orlando eine Oper mit komischen Elementen, die mit der Wahnsinnsszene Orlandos und mit der Figur von Dorinda zusammenhängen. ${ }^{22}$ Dorinda ist eine Schäferin, die sich in einem niedrigen Stil ausdrückt, der eher zur tragikomischen Pastorale passen würde. Ihre Vokalität entspricht allerdings dem anspruchsvollen Koloraturgesangsstil des ernsten Dramma per Musica. Die Arie "Amor è qual vento" vertritt einen solchen gemischten Stil:

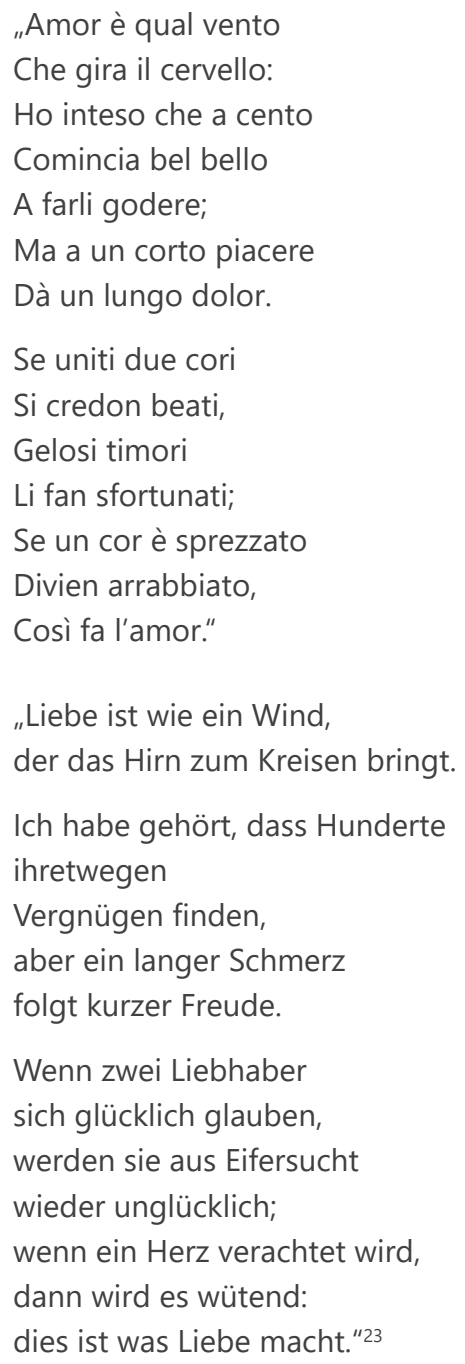

21 Dean, Handel's operas, S. 466.

22 Reinhard Strohm, "Comic traditions in Handel's Orlando", in: Essays on Handel and Italian Opera, Cambridge 2008, S. 249-270.

23 Übersetzung L.M. 
Der Text ist ungewöhnlich lang für eine Dacapo-Arie der Zeit und verwendet nicht nur Worte aus einem niedrigen Sprachregister ("cervello" statt "cerebro", "arrabbiato" statt "furente"), sondern auch umgangssprachliche Ausdrücke wie etwa "bel bello". Ein Held aus einem Libretto von Zeno oder Metastasio könnte sich kaum auf diese Weise äußern, Dorinda hingegen schon, weil sie eine Schäferin ist. Der Text würde eher zu einem Lustspiel passen, denn die Musik mischt eine sehr anspruchsvolle Vokalität mit den Elementen, die auch bei der Arie "Amante stravagante" aus Flavio auffällig sind: übertriebene Intervallsprünge und für Händels Kompositionen ungewöhnliche Wechselspiele zwischen Dur und Moll.
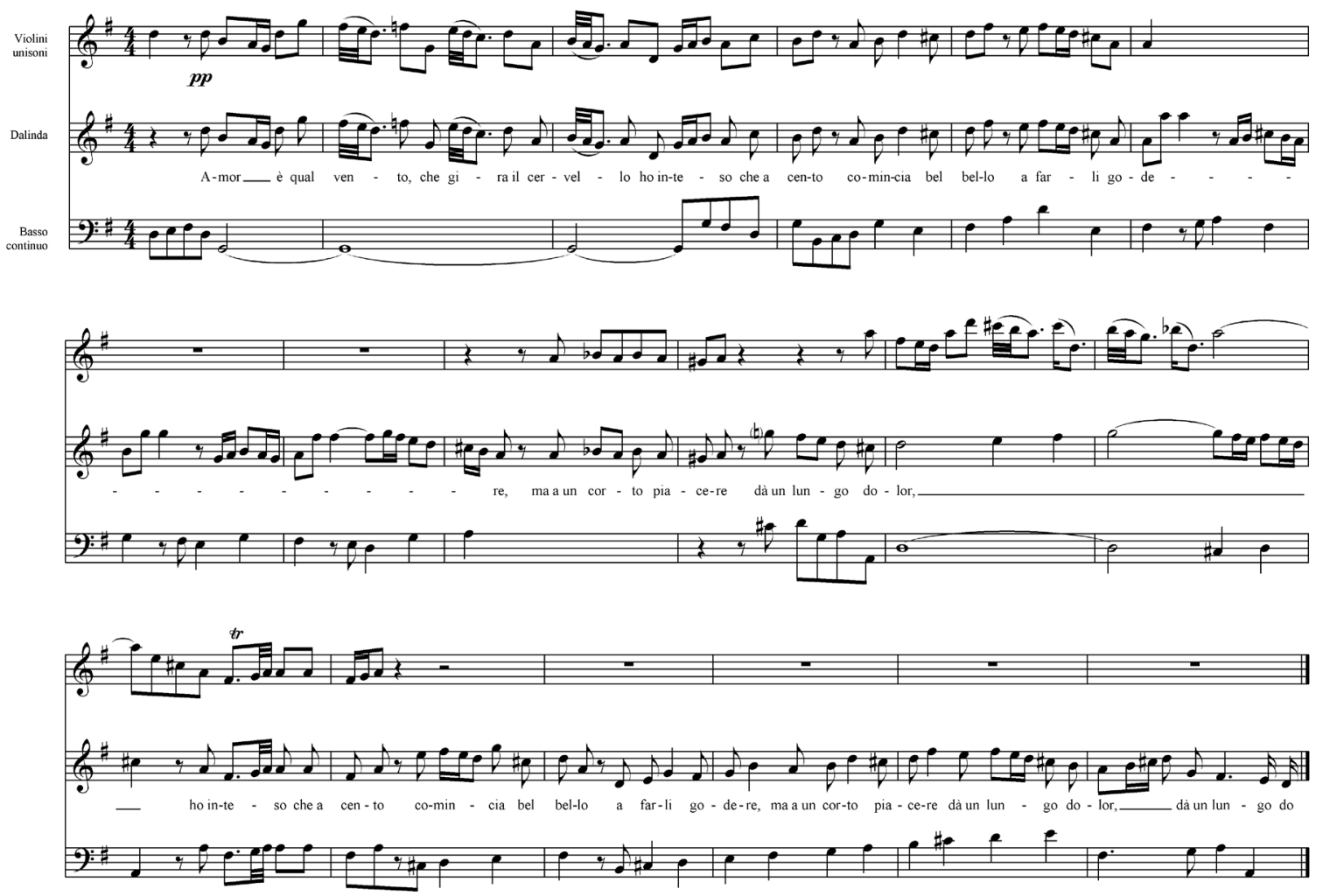

Notenbeispiel 6: Georg Friedrich Händel, „Amor è qual vento”, aus Orlando (HWV 31).

Die im vorliegenden Text ausgewählten Beispiele stellen einen Versuch dar, die dreigeteilte Kategorisierung Matthesons im Spiegel seiner zeitgenössischen Opernproduktion zu untersuchen und die heutzutage etablierte Dichotomie zwischen Opera seria und Opera buffa anhand seiner Definition eines Strafspiels infrage zu stellen. Statt einer pauschalen Verwendung der Kategorie des Komischen bei der Analyse dramatischer Vokalmusik unterschiedlicher Gattungen plädiere ich eher für eine Unterscheidung zwischen dem Komischen der Opera buffa und des Intermezzo sowie dem Satirischen gemischter Gattungen, dem die kompositorische Benutzung auffälliger stilistischer Inkongruenzen im Sinne einer "burlesken Travestie" zugrunde liegen. 
Zitation: Livio Marcaletti, „,Strafspiel' und satirische Stilmittel in musikdramatischen Gattungen des frühen 18. Jahrhunderts", in: Freie Beiträge zur Jahrestagung der Gesellschaft für Musikforschung 2019, hrsg. von Nina Jaeschke und Rebecca Grotjahn (= Musikwissenschaft: Aktuelle Perspektiven. Bericht über die Jahrestagung der Gesellschaft für Musikforschung 2019 in Paderborn und Detmold, Bd. 1), Detmold 2020, S. 211-223, DOI: 10.25366/2020.63. 


\section{Abstract}

The tendency of today's historiography to portray early 18th-century Italian opera as a dichotomy between opera seria and opera buffa takes too little account of the existence of genera mixta. However, contemporary composers and authors sometimes referred to a tripartiton. In his treatise Der vollkommene Capellmeister (1739), Johann Mattheson distinguishes between tragedy, comedy and satire. His description of the melodies from a satirical opera is limited to the statement that they are "ridiculous, poseuristic and prickly". This definition can be applied to the analysis of dramatic vocal works with the help of Gerard Genette's category of "burlesque travesty" which describes the stylistic degradation of a tragic-heroic subject as a satirical function. This stylistic mixture is achieved by the use of specific musical devices, which are shown in this article on the basis of case studies on music by Francesco Bartolomeo Conti, Johann Sebastian Bach and Georg Friedrich Händel.

\section{Kurzvita}

Livio Marcaletti, 1984 in Bergamo geboren, studierte Musikwissenschaft und Italianistik an der Universität Pavia (Italien) und promovierte 2015 an der Universität Bern (Schweiz). Daraufhin hat er, mittels zweier SNF-Mobilitätsstipendien, an der Universität Wien gemischte Operngattungen im frühen 18. Jahrhundert untersucht. Zurzeit beschäftigt er sich an der Österreichischen Akademie der Wissenschaften mit einem Forschungsprojekt über deutsche Opernübersetzungen im 17. und frühen 18. Jahrhundert. 


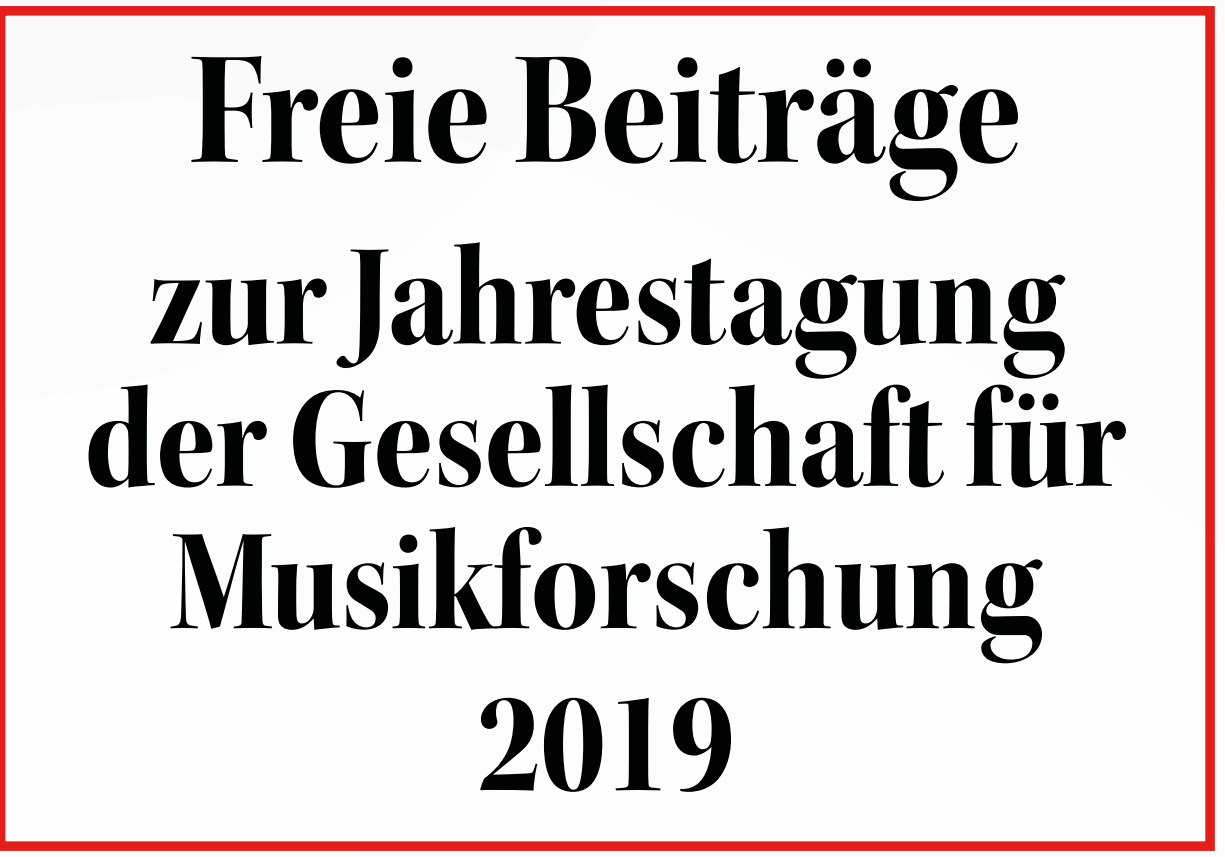

Herausgegeben von Nina Jaeschke und Rebecca Grotjahn

Musikwissenschaft: Aktuelle Perspektiven 1 
Freie Beiträge 


\section{Musikwissenschaft: Aktuelle Perspektiven}

Bericht über die Jahrestagung der Gesellschaft für Musikforschung 2019 in Paderborn und Detmold

Herausgegeben von Rebecca Grotjahn und Nina Jaeschke

Band 1 


\section{Freie Beiträge}

\section{zur Jahrestagung der Gesellschaft für Musikforschung 2019}

Herausgegeben von Nina Jaeschke und Rebecca Grotjahn

Detmold: Musikwissenschaftliches Seminar der Universität Paderborn und der Hochschule für Musik Detmold 2020 
DOI: $10.25366 / 2020.42$

Online-Version verfügbar unter der Lizenz: Urheberrecht 1.0, $<$ https://rightsstatements.org/page/InC/1.0/?language=de>

Bibliografische Information der Deutschen Nationalbibliothek

Die Deutsche Nationalbibliothek verzeichnet diese Publikation in der Deutschen Nationalbibliografie; detaillierte bibliografische Daten sind im Internet über http://dnb.d-nb.de abrufbar.

\section{Impressum}

Redaktion: Nina Jaeschke, Rebecca Grotjahn und Jonas Spieker Satz: Nina Jaeschke

(C) Musikwissenschaftliches Seminar der Universität Paderborn und der Hochschule für Musik Detmold 2020 


\section{INHALT}

Vorwort $\quad$ IX

Komponieren für das Radio: Akteure, Diskurse, Praktiken $\quad 1$

Musikwissenschaft - Feminismus - Kritik: Ein Generationenaustausch 6

\section{Stefan Alschner}

Der Wagner-Sänger Joseph Aloys Tichatschek - Vom Nachlass zum Netzwerk

\section{Alenka Barber-Kersovan}

Songs for the Goddess. Das popmusikalische Neo-Matriarchat zwischen Ethno-Beat,

erfundenen Traditionen und kommerzieller Vermarktung

Elias Berner, Julia Jaklin, Peter Provaznik, Matej Santi, Cornelia Szabó-Knotik

Musikgeschichte anders erzählen? Das Beispiel der 1970er in Österreich.

Musikhistoriographie in der Zeit der Digitalisierung

\section{Mauro Fosco Bertola}

„Ein Laut so klagevoll”. Lohengrin zwischen Richard Wagner und Salvatore Sciarrino

\section{Matthieu Cailliez}

Europäische Rezeption der Berliner Hofoper und Hofkapelle von 1842 bis 1849

\section{lacopo Cividini}

Zwischen klassischer Musikphilologie und angewandter Informatik:

Die Digitale Mozart-Edition (DME) der Stiftung Mozarteum Salzburg

\section{Marko Deisinger}

Fortschrittliche Technologie im Dienste eines Antimodernisten.

Heinrich Schenker und der österreichische Rundfunk

\section{Norbert Dubowy}

Vom Kritischen Bericht zur Kritischen Dokumentation am Beispiel der Digital-interaktiven Mozart-Edition

\section{Markus Engelhardt}

Musik zwischen Nation Building und Internationalität. Italien um 1900

\section{Maryam Haiawi}

Das Oratorium im Spannungsfeld der Konfessionen: 


\section{Judith I. Haug}

"Manch eine*r liegt, morgens noch trunken, im Rosengarten" - Rekonstruktionen

osmanischer Musikgeschichte in Gesangstextsammlungen

\section{Renate Koch}

Marcel Prawy und das erste Broadway-Musical im Österreich der Nachkriegszeit

Susanne Kogler, Julia Mair, Juliane Oberegger, Johanna Trummer

Erich Marckhl - Musikausbildung in der Steiermark nach 1945.

Brüche und Kontinuitäten

\section{Marie-Anne Kohl}

Die weinende Jury. "Geschlechtslose" Tränen bei globalen Musik-Castingshows?

\section{Fabian Kolb}

Tanztheater und filmische Ästhetik. Cineastische Einflüsse und Gestaltungsweisen in den Kompositionen für die Ballets Suédois 1920-1925

\section{Christian Lehmann}

Tempobezeichnungen von Julius Stockhausen für Die schöne Müllerin:

Ein Quellenfund

\section{Martin Link}

Signum et gens - Zur Gendersemiotik in Clara und Robert Schumanns Liederzyklus Liebesfrühling

\section{Livio Marcaletti}

„Strafspiel" und satirische Stilmittel in musikdramatischen Gattungen des frühen 18. Jahrhunderts

\section{Tobias Marx, Martin Lissner}

Thüringer Musikszene - Jugendmusikredaktionen als außerschulische musikbezogene Bildungskontexte

\section{Maho Naito}

Die Parallelität der Entstehungsprozesse der ersten beiden Symphonien Gustav

Mahlers: Instrumentation, Revision und Dirigierpraxis

\section{Elisa Novara}

Eine Schumann-Werkstatt? Zur Übertragbarkeit der Methoden vom Projekt 
Theodora Oancea, Joachim Pollmann, Jonas Spieker

Kollaborateure - Involvierte - Profiteure. Erarbeitung eines Online-Lexikons zur

Musik in der NS-Zeit

\section{Kiron Patka}

„Ich wollte eigentlich Sängerin werden." Berufsselbstbilder von Tontechniker*innen im Radio

\section{Siegwart Reichwald}

Die Leiden der jungen Clara: Das Klaviertrio Opus 17 als Ausdruck einer Neu-

Romantikerin

\section{Elisa Ringendahl}

Lied versus Oper - Pole musikalischer Gattungen bei Oscar Bie

\section{Benedikt Schubert}

Struktur und Exegese. Über Eigentümlichkeiten in der Arie "Des Vaters Stimme ließ sich hören" (BWV 7/4)

Uwe Seifert, Sebastian Klaßmann, Timo Varelmann, Nils Dahmen

Computational Thinking in der Musikwissenschaft: Jupyter Notebook als Umgebung

für Lehre und Forschung

\section{Yusuke Takamatsu}

Synthese als Modus der Prozessualität bei Schubert:

Sein spezifisches Wiederholungsprinzip im langsamen Satz

\section{Daniel Tiemeyer}

Johann Nepomuk Hummels Sonate in fis-Moll Op. 81 - Studien zu Entstehungs-

hintergrund, Rezeption und formalerStruktur

\section{Andrea van der Smissen}

Musikalische Innovation im Umfeld der Moderne und historischen Avantgarde in Ungarn

\section{Tim Ziemer, Holger Schultheis}

Psychoakustische Sonifikation zur Navigation in bildgeführter Chirurgie

\section{Magdalena Zorn}

Musik mit dem Radio hören: Über den Begriff der musikalischen Aufführung 
Gabriele Buschmeier in memoriam 


\section{Vorwort}

Die vorliegenden Bände dokumentieren die Jahrestagung der Gesellschaft für Musikforschung 2019. In den dreieinhalb Tagen vom 23. bis zum 26. September 2019 wurden in Paderborn und Detmold nicht weniger als 185 Beiträge präsentiert, verteilt auf diverse Symposien, Round tables, Freie Sektionen und Postersessions. Sie alle auf einen Nenner bringen zu wollen, ist ein Ding der Unmöglichkeit - und das ist gut so, ist es doch Ziel der Jahrestagungen, die große Vielfalt der Themen und Methoden des Faches Musikwissenschaft abzubilden. Um die thematische Vielfalt der freien Referate angemessen abbilden zu können und gleichzeitig den inhaltlichen Schwerpunkten der beiden hier publizierten Hauptsymposien ausreichend Raum bieten zu können, erscheinen diese in drei Bänden.

„Musikwissenschaft: Aktuelle Perspektiven": Der Titel der kleinen Reihe ist keine Verlegenheitslösung. Musikwissenschaft im Kontext der Digital Humanities; Musikwissenschaft und Feminismus; Musik und Medien; Musikalische Interpretation - schon die Felder, die von den vier Hauptsymposien bespielt wurden, wären noch vor wenigen Jahrzehnten allenfalls an der Peripherie das Faches zu finden gewesen. Sie entsprechen Arbeitsschwerpunkten der Lehrenden am Musikwissenschaftlichen Seminar der Universität Paderborn und der Hochschule für Musik Detmold, das die Tagung ausrichtete. Zugleich nehmen sie Bezug auf aktuelle Ereignisse und Entwicklungen. So erwuchs das von Andreas Münzmay und Joachim Veit organisierte Symposium „Brückenschläge - Informatik und Musikwissenschaft im Dialog" unmittelbar aus den Erfahrungen im Virtuellen Forschungsverbund Edirom (ViFE) und im fakultäten- und hochschulübergreifenden Zentrum Musik-Edition-Medien (ZenMEM). Der 200. Geburtstag von Clara Wieck/Schumann war der Anlass für das von Rebecca Grotjahn geleitete Symposium „Die Begleiterin - Clara Schumann, Lied und Liedinterpretation", das in enger Kooperation mit der Hochschule für Musik Detmold durchgeführt wurde. Das Hauptsymposium „Brückenschläge" wird in einem separaten Band publiziert (Bd. 3 der vorliegenden Reihe). Im Rahmen dieses Symposiums führte die von Stefanie Acquavella-Rauch geleitete Fachgruppe Digitale Musikwissenschaft eine Posterpräsentation durch, die von den Beiträger*innen erfreulicherweise zu kürzeren Texten umgearbeitet wurden, sodass sie hier ebenfalls, zusammen mit den Postern,

publiziert werden können. Hinzu kommen einige Beiträge, die bereits bei der Jahrestagung 2018 in Osnabrück präsentiert wurden. Auch das Hauptsymposium "Die Begleiterin" wird in einem eigenen Band (Bd. 2) publiziert. Die Beiträge zu den beiden anderen Hauptsymposien hingegen werden an anderen Orten veröffentlicht; in Band 1 („Freie Beiträge zur Jahrestagung der Gesellschaft für Musikforschung 2019") der vorliegenden Publikation finden sich jedoch Einführungen und Abstracts. Das Symposium „Komponieren für das Radio" unter Leitung von Antje Tumat und Camilla Bork (Katholieke Universiteit Leuven) behandelte Einflüsse des Mediums auf Kompositionsprozesse sowie durch radiophone Kompositionen bzw. radiophonen Klang ausgelöste Diskurse. Sarah Schauberger und Cornelia Bartsch (Universität Oldenburg) nahmen das 25-jährige Jubiläum der Fachgruppe Frauen- und Genderstudien zum Anlass für einen Generationenaustausch zum Thema "Musikwissenschaft - Feminismus - Kritik": Was wa- 
ren vor einem Vierteljahrhundert Inhalte und Aufgaben einer feministischen Musikwissenschaft und wie kann sich diese heute positionieren?

Bewusst haben wir im Tagungsbericht auf inhaltliche Eingriffe in die Beiträge verzichtet. ${ }^{1}$ Das gilt besonders für die Freien Referate: Es galt, den Charakter der Jahrestagung als Forum für ,freie', d. h. innovative und auch experimentelle Gedanken zu wahren. Einige Kolleg*innen, die die Tagung mit Vorträgen und Posterpräsentationen bereichert hatten, haben sich gegen eine Publikation im vorliegenden Band entschieden - sei es, weil sie eine Möglichkeit fanden, ihre Beiträge in einem inhaltlich passenderen Rahmen zu veröffentlichen, sei es, weil ihre Überlegungen in ihre entstehenden Qualifikationsschriften fließen sollen, oder sei es, weil sie von den Autor*innen in der vorgetragenen Form zunächst verworfen wurden. Auch damit erfüllt eine Freie-Referate-Sektion ihren Zweck: Die Diskussionen mit der versammelten Fach-Öffentlichkeit sollen dabei helfen, Gedanken weiterzuentwickeln und zu verändern. In diesem Sinne sei allen Beteiligten - den Autor*innen, den nichtpublizierenden Referent*innen und den Mit-Diskutant*innen - ganz herzlich gedankt für ihr Mitwirken bei der Tagung.

Unser herzlicher Dank gilt einer Reihe weiterer Personen, die zum Gelingen dieser drei Bände beigetragen haben. Hier ist besonders Jonas Spieker zu nennen, der uns tatkräftig bei der Redaktion geholfen hat. Andrea Hammes (SLUB Dresden) sei herzlich für die Aufnahme unseres Bandes auf musiconn.publish gedankt - wir freuen uns, damit unsererseits zur Etablierung dieser innovativen Publikationsplattform beizutragen.

Erneut möchten wir an dieser Stelle allen Menschen danken, die uns bei der Organisation, Ausrichtung und Finanzierung der Tagung selbst unterstützt haben: der Präsidentin der Universität Paderborn, Prof. Dr. Birgitt Riegraf, dem Rektor der Hochschule für Musik Detmold, Prof. Dr. Thomas Grosse, den Kolleginnen und Kollegen der beiden beteiligten Hochschulen, dem Vorstand der Gesellschaft für Musikforschung, der Universitätsgesellschaft Paderborn und allen Sponsoren. Besonders dankbar sind wir den Mitarbeiter*innen und den studentischen bzw. wissenschaftlichen Hilfskräften des Musikwissenschaftlichen Seminars, die bei der Vorbereitung und Ausrichtung der Tagung immensen Einsatz zeigten - stellvertretend sei an dieser Stelle Johanna Imm erwähnt, die zusammen mit Nina Jaeschke das Herz des Organisationsteams bildete.

Wir widmen diese Reihe Dr. Gabriele Buschmeier, dem langjährigen Vorstandsmitglied der Gesellschaft für Musikforschung, die kurz vor der Publikation dieses Bandes unerwartet verstarb.

Detmold, im September 2020

Rebecca Grotjahn und Nina Jaeschke

Zitation: Rebecca Grotjahn und Nina Jaeschke, „Vorwort”, in: Freie Beiträge zur Jahrestagung der Gesellschaft für Musikforschung 2019, hrsg. von Nina Jaeschke und Rebecca Grotjahn (= Musikwissenschaft: Aktuelle Perspektiven. Bericht über die Jahrestagung der Gesellschaft für Musikforschung 2019 in Paderborn und Detmold, Bd. 1), Detmold 2020, S. IX-X, DOI: 10.25366/2020.43.

1 Freigestellt war den Autor*innen auch, ob sie sich für eine gendersensible Sprache entscheiden bzw. welche Form des Genderns sie bevorzugen. 